\section{A $\mathfrak{R}_{\mathfrak{o r}} \mathfrak{p} \mathfrak{n} \mathfrak{r t}$}

CASES ILLUSTRATING THE AID OF THE ROENTGEN RAYS IN THE DIAGNOSIS OF INTRA-

\section{THORACIC TUMOURS.*}

\section{BY}

J. MAGEE FINNY, and EDWARD J.M. WATSON, M.D.Dubl.,

Ex-President R.C.P.I. ; King's Professor of Practice of Medicine; Patrick Dun's Hospital.

B.A.Cantab., M.D.Dubl., Officer in Charge of the $X$ Ray Hospital.

The aocompanying illustrations are reduced fiom the skiagraphs of the cases, and I have added for the sake of comparison a skiagraph of a normal thorax.

Case i.-Sarcoma of Right Lung.

C. B., aged 30 a dressmaker was admitted under my care to Sir Patrick Dun's Hospital on the recommendation of Dr. Wright, Dalkey, on February 29th, 1901. Dr. Wright had treated her for haemoptysis, and recognizing that her eymptoms were of an unusual character, advised her being placed under observation and treatment in hospital.

THE diagnosis of intrathoracic disease, it will be readily acknowledged by every practising and consulting physician, is at all times a matter of clinical acumen; and although it may be arrived at in some cases with comparative ease and by the ordinary methods of clinical examination, in other

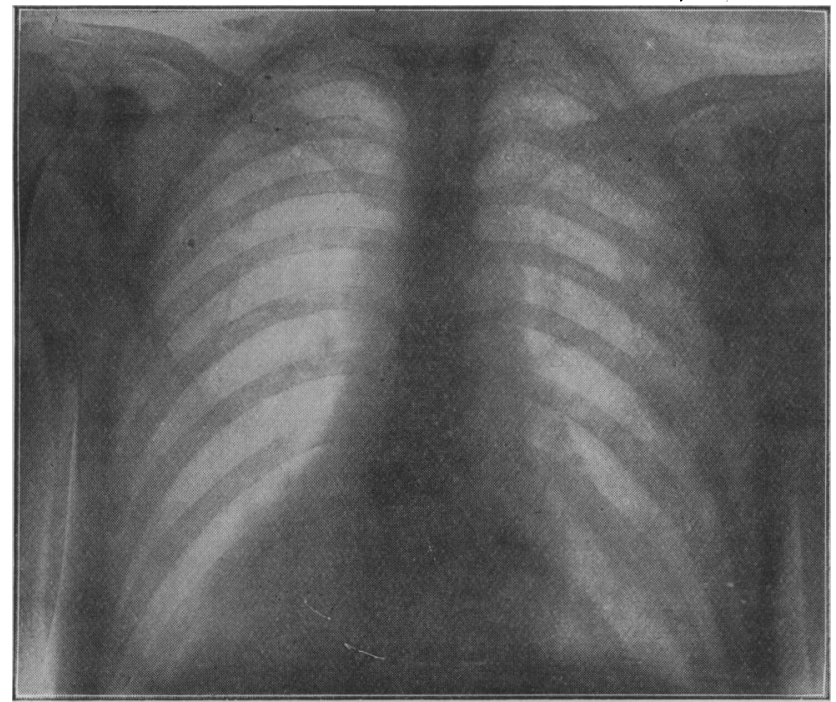

Normal thorax. Female, aged 34. (Taken from behind.)

cases, and especially in those of suspected intrathoracic tumours, it is often one of the most difficult problems presented to the physician, and may be impossible by those same tests.

Of course there are instances of aneurysms of the arch of the aorta or of malignant disease of the lungs which can be recognized as such with any ordinary care and observation; but even then, while the presence of a new growth or an arterial dilatation is readily made out, the extent and size of an aneurysmal sac or the exact site of a cancer must be at best but a question of probability or a bare guess. Then again at times an aneurysm may present no physical signs whatever to betray its presence, while, on the other hand, a mediastinal neoplasm may so closely simulate an aneurysm in its symptoms as to defy differentiation.

Any aid, therefore, which can help to clear up such difficul ties and remove such doubts, and which can chinge deductive evidence into demonstrable must be gladly welcomed.

Such an aid, I believe, we may have in the $x$ rays, and I would like to illustrate this point in the following cases, which occurred in my practice during the year rgor. Two are cases of aneurgsm, and one of malignant disease occurring within the chest. In all the $x$ rays were a help more or less in removing doubts and in explaining anomalous symptoms, while in one it was the only means I had of detecting an aneurysm of the thoracic aorta.

And here I gladly acknowledge the able assistance I got from Dr. Edward Watson in the examination of these cases by means of both the screen and the radiograph.

* Read before the Royal Academy of Medicine in Ireland, March 7th, 1902.

\section{History.}

Her history was indefinite, but it appeared that she had not felt well for three or four months, and had lost flesh. About six weeks ago she had ome slight pains in her right side, and had a cough and had spat up a blood. At the same time she noticed that her right breast was large than the left, and that the right side of her chest appeared swollen. There is no history of any previous illness.

\section{Present Conditzon.}

She has very little cough or any distress except breathlessness on exertion normal: pulse, 100; respirations, 24

Inspection. - The patient appear's well nourished. Her face is, as a rule, of a good colour, but sometimes shows a cyanotic hue, and there is a decided puffiness under the eyes, and both sides are swollen. The jugulars are prominent, and the right supraclavicular fossa is swelled The infraclavicular region is also puffed out with a frm semi-elastic infiltration of the skin or subcutaneous tissues, which does not pit on pressure. The right side of the thorax is considerably larger than the left-by 2 Inches-both above and below the mamma. Th not nodular, the heavy and large, and feels hard and inelastic in the gland. The superswelling being in the areolar tissue rather than in the gland. The superficial veins over the thorax are enlarged, and at tle level of the diaphragm extend to the left side. The current of the stream is the left.

ight arm measures three-quarters of an inch more than the left. fremitus could not be established; the feel of the whole side is that of induration and thickening, so that the ribs cannot be readily made out.

Percussion -Above the right clavicle a high-pitched note is obtainable below the clavicle the whole of the front of the right chest is dull, and the dullness clavicle the wack a highdulness extends across heard above a line drawn from the ninth dorsal pitched note is to be process; all below that line a flat non-resonant note. Change of position made no alteration in these signs.

A uscultation - Over the affected side the breath sounds are practicall annulled, vocal resonance being diminished below and rather incre sed above.

Circulatory System.-The heart is normal in position and sounds. The pulse is rapid, circulation roo, and equal in both radials. The urine is normal.

As the diagnosis inclined to the existence of an intrathoracic growth involving the intercostal veins and superior cava, Dr. Edward J. Watson (the officer in charge of the $x$-ray department at the hospital) took a skiagraph. The result was not, however, satisfactory, as the whole side was more or less almost uniformly opaque, such as might be due to pleural effusion alone. Yet on looking closely at the picture, an ill-

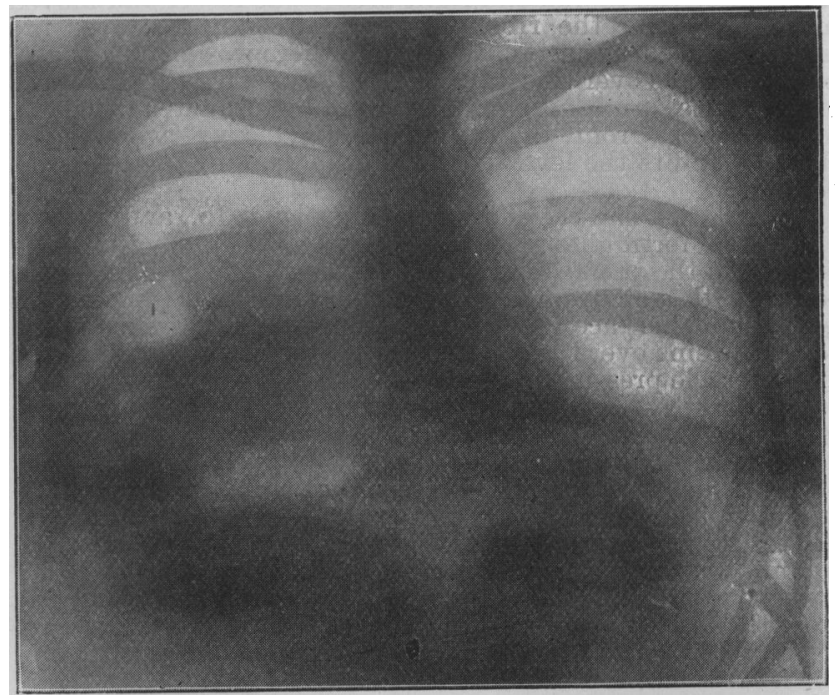

Case I.-C. B. Sarcoma of the right lung (taken in front).

defined mass, corresponding to the deeper shadow in the lowe: half, could be faintly outlined. 
An exploratory puncture was made on April 5 th, and some clear yellow fluid drawn off. It was then plain that there was, as expected, fluid in the pleura. Thoracocentesis was done on April 8th, when $72 \mathrm{oz}$. were obtained by siphon drainage; the first was yellow and clear serum, the latter third was blood-stained. The fluid did not coagulate on standing and presented no inflammatory corpuscles or morbid cells.

The interest now lay in the physical signs. After the tapping the air was found to enter the upper part of the lung, and to a less extent the lower. The dull note disappeared over the whole back and axillary regions, and in front down to he fourth rib; below this it remained dull. This dullness might have been due to an encysted pleurisy, which had not communicated with the posterior effusion, and had thus escaped drainage, or, as I strongly suspected, it was due to a neoplasm which by its pressure caused the oedema of the right thorax and the varicosity of the superficial veins. Besides, a pleural effusion alone could not explain the jugular and cervical distension.

For a couple of days after the tapping improvement was experienced, the swelling of face and neck diminished, and

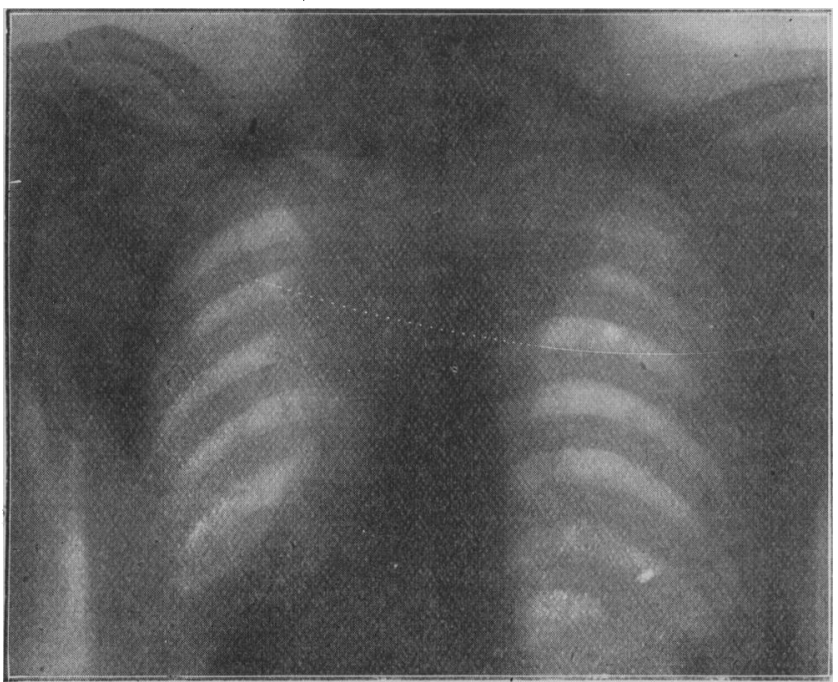

Case 11.-P. C., aneurysm of the arch of the aorta (taken from behind).

the skin of the thorax and right breast was looser and moved more freely over the ribs. However, the dullness over the front of the chest never altered, and there was an absence of breath sounds over this dull area. On the $13^{\text {th }}$ the fluid was evidently re-forming, as in addition to the dull area below the fourth rib in front, the dullness extended up again to the clavicle, and to the level of the inferior angle of the scapula behind.

April 14th.-The patient weighs but 6st. II lb., and has lost $3 \mathrm{lb}$. in a fortnight. She has been up each day since the tapping of the 5 th feeling greatly relieved, and thinks herself better, although from the returning and more persistent cyanosis of her face and lips, the increase in the size of the varicose veins over the thorax and abdomen, it is evident the intrathoracic pressure is not relieved.

April 23rd.-A second tapping was done, and $62 \mathrm{oz}$. were removed; the fluid was straw-coloured, with a few flocculi of fibrin in it. It did not spontaneously coagulate.

On the day following Dr. Watson took another $x$-ray photograph, which demonstrated clearly the presence of a tumour (Plate II) in front of and to the right side of the vertebral column, extending from the level of the fourth rib behind, down to a short distance above the diaphragm, and occupying more than three-quarters of the lung space, and having regard to the persistent dullness in front, it presumably lies nearer the front than the back of the thorax.

During the months of May and June, three weeks of which she spent at home, there was little change, but the fluid slowly re-formed, and the swelling in the breast, etc., returned. She was readmitted on July ist, with an aggravation of all her symptoms and, in addition, there were much insomnia due to orthopnoea, and some difficulty in swallowing. The physical signs of cutaneous oedema of eyelids, face, neck, and thorax, as well as those of the lungs and respiration, were much as before the first tapping; yet, in the hope that some relief might be obtained, she was tapped a third time, when $160 \%$ were drawn off.

No relief to her distress, and no change in the physical signs followed it. The only ease the patient got was from the hypodermics of morphine. She left hospital a week later, and died at home on July I8th, in an attack of dyspnoea-the second after her return.

REMARKS.

There are some features in the foregoing case worthy of note.

The onset of the disease, which lasted about eight months, was insidious. It was not preceded by any operative proceeding for external cancer, nor by evidences of disease in other regions or organs. The intrathoracic tumour is, therefore, the primary disease, and although not positively proved, owing to the impossibility of obtaining a necropsy, yet considering the age of the patient, its nature in all probability was sarcomatous, and its origin in the connective tissue of glands of the posterior mediastinum, from which its growth spread peripherally and laterally to the right, and invaded the pleura and lung. Its pressure on the intercostal veins caused the oedema over the right half of the thorax, and it further involved the vena azygos and its tributary, the superior intercostal, as it reached the superior vena cava. It is probable it pressed also upon the superior cava itself or the right innominate vein.

The cyanosis, the swelling in the supraclavicular fossae, and the varicosity and large size of the superficial veins of the thorax and abdomen, in which the direction of the blood stream was downwards, could not well be otherwise explained.

Case iI.--Aneurysm of the Arch.

P. C., farmer, aged 42, married, was admitted to Sir Patrick Dun's Hospital on March 2rst, rgor, under my care, with an aneurysm of the thoracic aorta, on the recommendation of Dr. John G. Gibbon, of Mullingar, co. West Meath.

History.
The patient states that he has been for many years in the habit of drinking a large quantity of whisky-up to 13 glasses a day: that about eighteen months ago he suffered from pain and a feeling of stiffness along the right side of the neck, and at the same time he noticed a lump at the upper part of the right side of the chest in front. He consulted a physician, and was given medicine, with the result that the pain left him in three or four days: that for eight months subsequently he was free from trouble, and that then, about three months ago. the pain began to trouble him again, being at times unbearable in the right shoulder. He saw the same physician again, and being no better, was treated by Dr. Gibbon, who gave him potassium iodide gr. $x \nabla$ thrice daily with great relief. He

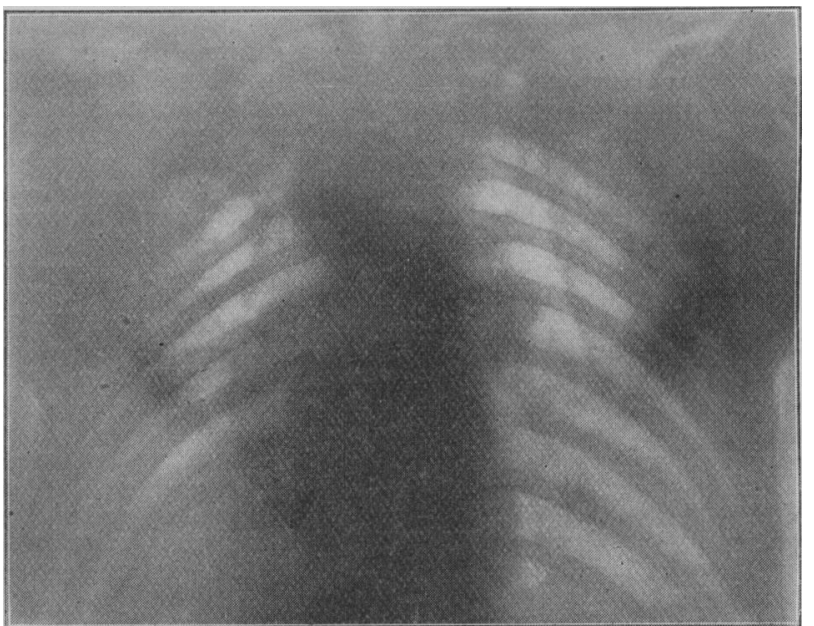

Case III.-D. M., aneurysm of the aorta (taken from behind). also had morphine administered hypodermically for the pains. Patient states that fourteen zears ago he suffered from numbness and pain in his states that fourteen years ago he suffered from numbness and pain in his
left hand and arm, that his hand used to get white and cold at intervals 
and he lost complete power over it. On consulting a doctor he was told that his left radial pulse was much smaller than his right.

A month ago his cough became considerable, and ho brought up each day about a teacupful of "phlegm " mixed with some dark-coloured clotted blood Tubercle bacilli were not present in the sputum.

\section{Present Condition.}

The patient at present complains of pain more or less constant in the right shoulder and side of the neck; the pain varies in intensity, and at times becomes very acute.

$$
\text { Inspection and Palpation: Percussion. }
$$

There is a tumour present in the upper part of the right chest at the position of the sternal end of the clavicle and the attachment of the costal cartilages of the first and second ribs, and percussion dullness present in this situation for an area of 2 inches diameter. Expansive pulsa作 coft murmur synchronous with the cardiad to the there is a loud systolic murmur audible, and most distinctly toward the apex The right radial pulse is normal, the left is absent, and the brachial pulse cannot be felt.

Respiratory Sustem

Respiratry System.-The inspiratory murmur in the upper part of the right lung is delayed, and then enters with a rush, though feeble all over the right side. The air enters the left lung well above, but below and also through the lower lobe the sounds are masked by coarse crackling rales. rine, specific gravity to3o, reaction acid, no albumen.

April 3rd.-Dr. Watson took a skiagraph (see illustration), which graphically depicts the aneurysm of the ascending transverse and descending portions of the arch.

The patient was put on modified Tufnell's treatment, and he was given gr. $\times x \times$ of potassium iodide in the day, rest in horizontal positıon being strictly enforced, and all stimulants were withdrawn.

April 12th.-Patient complained of pain at night over the region of the aneurysm; he, however, slept well, but on waking seemed to have some difficulty in breathing. The dyspnoea became so much worse a few hours later, that he could not maintain the recumbent posture, but sat up leaning forward; stridor also ensued, with a hacking, harsh cough.

For two days he seemed in imminent danger of suffocation, tha only relief being given by medicated steam inhalations anc ushe hypodermic use of morphine in repeated doses.

Our senior surgeon, Dr. E. H. Bennett, saw him in consultation on the question of tracheotomy, but as the dyspnoea etc., were not due to direct laryngeal oedema or pressure, but rather to deep-seated pressure on the bronchus, it was discarded as useless. Fortunately the necessity did not arise, for in three days the aneurysmal pressure had varied in its direction, and the urgent symptoms abated and were quite gone in seven days.

The postural treatment, having been so interrupted on 12 th6th, was given up. There was now no difficulty in breathing or swallowing. The aneurysm appears to have receded somewhat from the anterior chest wall, the pulsation above the clavicle being still very well marked, but below the clavicle much diminished.

May 3rd.-Allowed up to-day, slight pain complained of behind the right ear and right side of the neck, which feels hotter than the left. The inner end of the right clavicle still pulsates with the beat of the aneurysm ; there is no discoloration of the skin over the tumour. The patient went out of hospital on May roth. The temperature was never above normal; the pulse ranged from 60 to 104 .

Recent inquiries are to the effect that the patient was alive and about in December last, and was able to take exercise, and that he had had no serious return of pain or dyspnoea. It is further reported that he had not continued the total abstinence which his seven weeks' residence in hospital had enforced and inculcated.

REMARKS.

In the foregoing case the interest is much enhanced by the location of the aneurysm by the $x$ rays. While it was evident from the ordinary clinical tests that aneurysm of the first portion of the arch of the aorta existed, and pressed forward at the usual place, and also at the sterno-clavicular joint, and that it extended into the transverse portion of the arch, and bulged up into the neck, pushing the trachea to the left, and engaging the opening of the left subclavian artery, it was not at all evident that the aneurysm was of the large size the skiagraph depicted, or that it extended quite as much to the left as to the right of the middle line, or that it reached from the level of the second to the sixth rib on the left, and of the first to the fourth on the right side.
Thus by this clinical aid it was demonstrated that the aneurysm extends into the third stage, and by its pressure against the left bronchus, as I imagine it did, it produced the symptoms of the haemoptysis, the mucopurulent expectoration, and the congestion of the left lower lobe in the beginning of his illness, and induced the dangerous attack of "stridor from below" (Stokes), and the dyspnoea of the latter stages of his case while in hospital.

It is to be regretted that a more recent skiagraph could not be obtained to define the present size and shape of the aneurysm, for, as it is evident that improvement has taken place, and its pressure signs and symptoms have proportionately diminished, it is more than probable that the sac has more or less contracted and become consolidated.

Case iit.-Aneurysm of Descending Aorta.

D. M., aged 54, admitted November 23rd, 100I, under my care. The patient is a flour miller, and had often to lift $20 \mathrm{st}$. sacks on his shoulder.

\section{History.}

He came to hospital complaining of pain localised in the left infra clavicular and supramammary regions. This pain was first noted about three weeks ago, while the patient was engaged in work of a fairly heavy fairly good, but six years ago he was operated on for a loft has always bee four years ago he got a tumour in the right inguinal region, for which the right testicle had to be removed. On inquiry at the Adelaide Hospital this tumour was said to be of an adenomatous type. 'The patient gives a definite history of having received an injury to the left testicle over two years ago, and as a result it became very painful, and after some time enlarged in which condition it remains.

He states that he has had gonorrhoea, but never syphilis. He states that he has lost $6 \mathrm{lb}$. in the last six weeks; his present weight is 8 st. $x_{2}^{2} \mathrm{lb}$. The pain is described as of a dragging character, shooting down into the left axilla and arm, and the pain in the arm is accompanied by a certain amount of numbness.

The pain appears to get worse when the patient is walking about or working, and he has had to stand or sit down in the street or at wor when thus affected, and this rest relieves him only for the time being and it has been bad enough to prevent his sleeping.

\section{Condition on Admission.}

Full and complete physical examination of the thorax failed to give the slightestindication of any disease of heart or blood vessels, lungs or pleura and there were no evidences of pressure on the veins or nerves, The only alteration discoverable was that the left side of the thorax was half an inch smaller than the right above and below the mamma. The cervical and supraclavicular glands were not enlarged. There was no slowing or alteration in the volume of the left radial pulse, and the pupils were

Under these negative signs I determined to seek the aid of the $x$ rays, and Dr. Watson examined the patient by the screen and made out a distinct area of shadow in the region of the descending aorta, and further noticed that it pulsated synchronously with the heart. He then took the skiagraph I exhibit (see p. 634, col. 2). The aneurysm is therefore of the descending aorta, and extends vertically from the third to the fifth ribs, and laterally for $2 \frac{1}{2}$ inches from the vertebral middle line opposite the fourth rib.

The treatment was based, even before the skiagraph, on the suspicion of an aneurysm and on the fact that staying in bed and lying down gave great relief from pain. It consisted in keeping him in bed for four weeks and administering potassium iodide gr. $v$ twice a day and ten grains at bedtime. His diet was not rigidly restricted. The temperature had been subnormal throughout. For a week before leaving hospital, which he did on January 17 th, 1902, he was free from all pains and felt better than he had done for months before admission in the last week of November, rgor. One day he walked for two hours without any recurrence of pain. He had gained $5 \mathrm{lb}$. while in hospital.

In this case there was a complete absence of all objective phenomena, and the only subjective symptom on which to lay stress was the existence of an ill-defined pain in the left thorax in front, referable to the third rib in the mammary line, and occasionally shooting out to the shoulder.

Now, though the presence of pain as the essential symptom of aneurysm should never be overlooked nor made little of it must be readily admitted that this symptom is not, in and by itself, sufficiently characteristic to justify a diagnosis of aneurysm. One is well aware that some patients may, with a moral obliquity and for a certain unworthy purpose, make great stock out of these pains; but, on the other hand, when there is no desire or intention to deceive, cases have occurred which have been made little of by the patient and the doctor alike, 
and yet have ended fatally by the sudden bursting of an intrathoracic aneurysm. In such cases the aid of the $x$ rays is incalculable, as it converts the possibility of an intrathoracic tumour being the cause of the pain into a certainty.

This it did in my case, where it might be called a revelation, and it did more, for it told me that the tumour was a pulsating one, expanding laterally at each cardiac beat. Of this particular feature Dr. Watson assures me he is as certain as that the heart itself moves with each ventricular systole and diastole. By the use of the screen this is more readily made out, and indeed the print of the photograph (exhibited) shows it as an indistinct and hazy halo surrounding the tumour at its side.

\section{POSTURE AND HEART MURMURS.}

BY W. GORDON, M.A., M.D., M.R.C.P.,

Physician to the Royal Devon and Exeter Hospital and the West of England Eye Infirmary.

VERY little attention has been directed to the influence of posture on heart murmurs. Murmurs are constantly being described and discussed without reference to the posture of the patient, the stray allusions in the textbooks (if we except the rather fuller treatment of " haemic " murmurs) are of the briefest sort, and statements so extreme as Potain's on "cardio-pulmonary " murmurs" are quoted without comment. The present position of the subject is thus summed up by Dr. Gee:

Some murmurs, which are audible whilst a patient is lying down, become much less loud or even disappear when he sits or stands. This is especially the case with mitral and tricuspid murmurs. The reason of the occurrence is not understood 2

I shall endeavour to show that the subject deserves more attention than has been bestowed upon it, and that there is an explanation of the phenomena which may be reasonably put forward.

If any one who has a considerable number of cardiac cases and cases of anaemia coming under his notice takes the trouble to examine these eases systematically both. in the recumbent and sitting postures, he will find that change from one to the other position produces effects on the cardiac sounds which are generally obvious and sometimes profound. In making such observations there are several sources of error which have to be avoided.

Infuence of Exertion.-To get rid of this the change of position must be effected by an assistant, so that no effort is made by the patient himself.

The Transitory Effect of the Change of Conditions.-A few pulse beats should be allowed to pass after changing the posture before listening again to the heart.

Possible Infuence of Altered Adjustment of the Stethoscope.The observations should be made with a straight wooden stethoscope.

"Cardio-pulmonary" Murmurs.-These should be excluded. Their dependence on respiration, their limitation to an area overlying the edge of the lung, and the fact that they do not follow the lines of conduction which characterize intracardiac murmurs should suffice to distinguish them. Potain's sweeping statement (that all murmurs which almost or altogether disappear when a change is made either from the sitting to the recumbent, or from the recumbent to the sitting posture are cardio-pulmonary) is not in accordance with the facts.

Having avoided these errors, it will be found that, although most murmurs are better heard when the patient lies down than when he sits up, this is by no means universal. There are murmurs which are uniformly influenced in the opposite direction, and there are murmurs in which position produces practically no 'effect. At first sight these differences seem merely bewildering, but on consideration it will, I think, be discovered that, instead of hindering, they actually help us to arrive at an explanation of the whole series of phenomena. In order to make this clear, I will briefly consider the effect of posture on each sort of murmur separately, confining the consideration to the commonest murmurs and to the two commonest positions, of sitting (or standing) and lying down.

$$
\text { A.- " Haemic" Murmurs. }
$$

1. In the Pulmonary Area.-The systolic murmur heard over the pulmonary artery is the murmur whose variations with varying posture have been definitely insisted on. Dr. Sansom says it is "greatly influenced" by the position of the body, being louder as the patient returns to the recumbent posture. ${ }^{3}$ This murmur is often markedly increased by lying down, and a murmur may even be developed which was inaudible in the erect position. Moreover, the area of audibility may be greatly extended; a murmur which is heard over quite a small area while the patient sits may become audible over an area three or four times the size when she lies down.

2. At the Apex.-The systolic apical murmur is also greatly influenced by posture, both in loudness and area of audibility. Fig. I illustrates the increase in area.

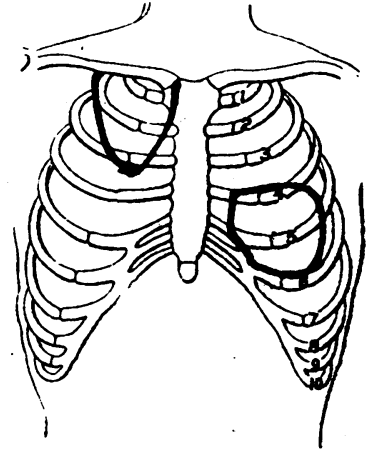

Erect.
Fig. r.-E. B. Two " haemic" murmurs fusing on recumbency

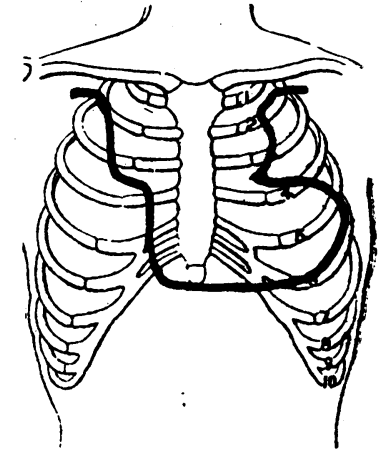

Erect.
Fig. x.-E. B. Two " haemic" murmurs fusing on recumbency 3. In the Aortic Area.-Fig. I also illustrates the fusion of two systolic " haemic" murmurs, one apical, the other "aortic," in consequence of each becoming audible over a wider area when the patient lies down. Fig. 2 similarly

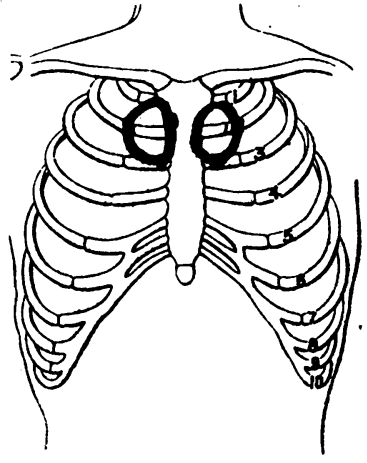

Erect.

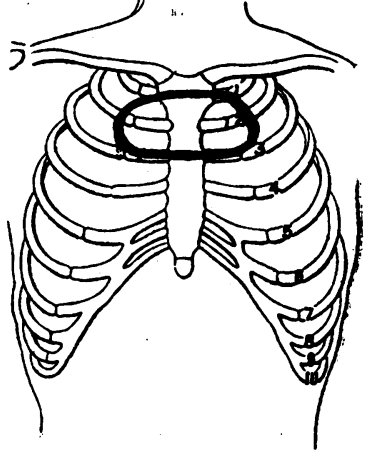

Recumbent.
Fig. 2-Fusion of two " haemic " murmurs on recumbency. illustrates the fusion of a "pulmonary" with an "aortic" haemic murmur.

4. Venous Hum in the Neck.-Although this is not a cardiac murmur it is necessary to refer to it here. It has been said

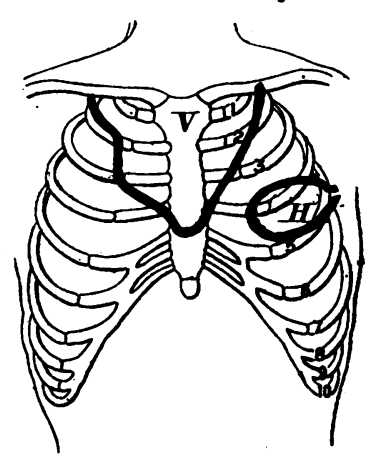

Erect.

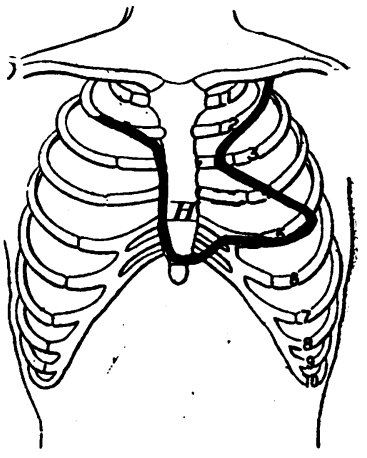

Recumbent.
Fig. 3. E. D. Chlorosis. The venous hum vanished on recumbency $v$, area of venous hum; $\mathbf{H}$, area of "haemic" murmur. 